\title{
G

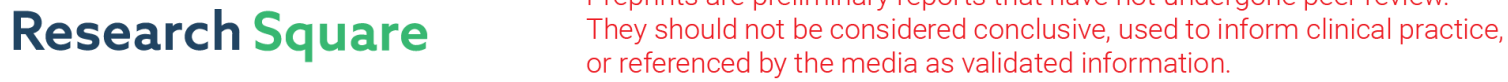 \\ Delta infection following vaccination elicits potent neutralizing immunity against the SARS-CoV-2 Omicron
}

\section{Mai-Juan Ma ( $\square$ mjma@163.com )}

State Key Laboratory of Pathogen and Biosecurity, Beijing Institute of Microbiology and Epidemiology https://orcid.org/0000-0002-8105-462X

\section{Lin Yao}

State Key Laboratory of Pathogen and Biosecurity, Beijing Institute of Microbiology and Epidemiology

\section{Hui-Xia Gao}

The Fifth Hospital of Shijiazhuang, Hebei Medical University, Shijiazhuang, China

\section{Ka-Li Zhu}

State Key Laboratory of Pathogen and Biosecurity, Beijing Institute of Microbiology and Epidemiology

\section{Jun Rong}

The Fifth Hospital of Shijiazhuang, Hebei Medical University, Shijiazhuang, China

\section{Li Yang}

The Fifth Hospital of Shijiazhuang, Hebei Medical University, Shijiazhuang, China

\section{Zhi Zhang}

The Fifth Hospital of Shijiazhuang, Hebei Medical University, Shijiazhuang, China

\section{Ping Jiang}

The Fifth Hospital of Shijiazhuang, Hebei Medical University, Shijiazhuang, China

\section{Li-Jun Duan}

State Key Laboratory of Pathogen and Biosecurity, Beijing Institute of Microbiology and Epidemiology

\section{Guo-Lin Wang}

State Key Laboratory of Pathogen and Biosecurity, Beijing Institute of Microbiology and Epidemiology https://orcid.org/0000-0002-5393-9500

\section{Er-Hei Dai}

The Fifth Hospital of Shijiazhuang, Hebei Medical University

\section{Article}

Keywords: SARS-CoV-2, Delta infection, Omicron, Vaccination, neutralization

Posted Date: January 10th, 2022

DOI: https://doi.org/10.21203/rs.3.rs-1245589/v1 
License: (c) (i) This work is licensed under a Creative Commons Attribution 4.0 International License. Read Full License 


\section{Abstract}

Since the initial detection of the severe acute respiratory syndrome coronavirus 2 (SARS-CoV-2) Omicron variant (B.1.1.529) in November 2021 in South Africa, it has caused a rapid increase in infections globally. The Omicron variant encodes 37 amino acid substitutions in its spike protein, and early reports have provided evidence for extensive immune escape and reduced vaccine effectiveness. We assessed serum neutralizing activity in sera from Delta infection following vaccination of CoronaVac or ZF2001 and Delta infection only against SARS-CoV-2 Wuhan-Hu-1 (WA1), Beta, Delta, and Omicron. We found that sera from Delta infection only could neutralize WA1 and Delta pseudoviruses but nearly completely lost capacity to neutralize Beta and Omicron pseudoviruses. However, Delta infection following vaccination resulted in a significant increase of serum neutralizing activity against WA1, Beta, and Omicron. This study demonstrates that breakthrough infection of Delta in previously vaccinated individuals substantially induced high potency humoral immune response against the Omicron variant and other emerged variants.

\section{Introduction}

Since the severe acute respiratory syndrome coronavirus 2 (SARS-CoV-2) was identified in late 2019, several SARS-CoV-2 variants of concern (VOC), including B.1.1.7 (Alpha) in the United Kingdom (UK), B.1.351 (Beta) in South Africa, P.1 (Gamma) in Brazil, and B.1.617.2 (Delta), have successively emerged with multiple substitutions in the spike glycoprotein. Among these four VOCs, the Beta variant showed the greatest immune evasion from serum neutralizing antibodies ${ }^{1}$ and compromised the efficacy of vaccines $^{2-4}$, whereas the Delta quickly outcompeted all other VOCs since first identified in October 2020 in India ${ }^{5}$ and partially escaped neutralization in vitro $^{6}$. The Omicron variant (B.1.1.529) was first detected in November 2021 in South Africa and has spread rapidly across the globe, outcompeting Delta within weeks to become the dominant circulating variant in several countries ${ }^{7}$. The large number of over 30 mutations in the spike protein of the Omicron resulted in extensively decreased the neutralization activity of certain monoclonal and serum polyclonal antibodies elicited by infection or vaccination ${ }^{8-13}$.

Before the emergence of the Omicron variant, high levels of Delta infections in many countries and reduced effectiveness of vaccines in preventing infection and transmission of the Delta variant ${ }^{14-17}$ might already result in tens of millions of infections in naïve or vaccinated individuals. In the face of rapid expansion of the Omicron infections worldwide and extensive escape from immunity elicited by vaccines and previous infection, understanding residual neutralizing activity in Delta infected individuals against the Omicron is essential to gauge the level of protection that a specific community has against infection, mild or severe COIVD-19. However, little is known about the susceptibility of Omicron to neutralizing antibodies elicited by Delta infection following vaccination and Delta infection only.

\section{Results}


To determine the susceptibility of the Omicron to Delta infection-induced serum activity, we analyzed convalescent serum samples obtained from 28 coronavirus disease 2019 (COVID-19) patients with the Delta infection only and 31 COVID-19 patients with the Delta infection following vaccination during the outbreak of the Delta variant between October and November 2021 in Shijiazhuang City, Hebei Province, China (Table 1). In addition, considering 26 of 28 COVID-19 patients with the Delta infection only from Shijiazhuang City were under 18 years old, convalescent serum samples from eight COVID-19 patients with the Delta infection only and $>18$ years old from a cargo ship were included for the analysis (Table 2). The median age for patients with the Delta infection following vaccination was 42 years (Interquartile range [IQR] 37-65; range 9-77 years), 10 years (IQR 8-12, range 2-68 years) for patients with Delta infection only from Shijiazhuang City, and 37 years (IQR 29-46; Range 19-50 years) for patients with Delta infection only from the cargo ship (Tables 1 and 2). Convalescent serum samples were collected at a median day of 47,38 , and 53 from symptoms onset to sampling for Delta infection following vaccination, Delta infection only from Shijiazhuang, and Delta infection only from the cargo ship. Among 31 patients with the Delta infection following vaccination, 22 have received two doses of CoronaVac, and 9 received three doses of the ZF2001 vaccine.

Because the results obtained in pseudovirus assays typically correlate well with those obtained against the authentic virus ${ }^{18}$. Neutralizing activity was measured using an established lentivirus-based pseudovirus assay. Sera were tested against pseudoviruses expressing the spike proteins of the WuhanHu-1 (WA1) vaccine strain and the Beta, Delta, and Omicron variants. We found that serum samples from Delta infection only, with a median day of 43 after infection to sampling, showed high levels of neutralizing activity against both the Delta and WA1 with a geometric mean titer (GMT) of 1245.0 (95\% confidence interval [Cl] 692.2-2240) and 563.0 (95\% Cl 300.3-1055.0), respectively (Fig. 1a). However, serum neutralizing activity against the Beta variant was decreased to a GMT of $38.5(95 \% \mathrm{Cl} 19.7-75.4)$, and only $15(41.7 \%)$ out of 36 serum samples displayed detectable serum neutralizing activity. Remarkably, only six (16.7\%) out of the 36 serum samples displayed detectable serum neutralizing activity against the Omicron, resulting in a GMT of $19.2(95 \% \mathrm{Cl} 11.4-32.5)$ with a 64.9 -fold decrease compared to the Delta variant (Fig. 1a). Further analysis showed no significant differences in antibody response between patients that > 18 years old and less than 18 years old regardless of WA1 and variants (Fig. 1b). These findings suggest that nearly all convalescent serum from patients with the Delta infection only loss the neutralizing activity to the Omicron, and antibody response was not affected by the age.

We next determined the neutralization activity of the serum from patients with the Delta infection following vaccination against WA1, Beta, Delta, and Omicron pseudoviruses. Interestingly, we observed that Delta infection following vaccination enhanced antibody response against WA1 with a GMT of 3942.0 (95\% Cl 2480.0-6265.0), which was significantly higher (3.4-fold) than antibody response against the Delta variant with a GMT of 1160.0 (95\% Cl 641.6-2098.0) (Fig. 1c). Moreover, although GMT against the Omicron variant displayed a 4.3-fold and 14.5-fold decrease compared to GMT against the Delta and WA1, respectively, the serum had a high potency to neutralize the Beta and Omicron variants. We observed that only one (3.2\%) and five (16.1\%) of 31 serum samples completely lost neutralizing activity 
against the Beta and Omicron variants, respectively (Fig. 1c). We then compared antibody response between patients vaccinated CoronaVac and ZF2001. No significant differences were observed for antibody response against WA1, Beta, and Omicron between the two groups, whereas CoronaVac vaccinated patients displayed a higher GMT of 1949 than ZF2001 vaccinated patients with a GMT of 960.4 (Fig. 1d). We further compared antibody response between patients with the Delta infection alone and patients with the Delta infection following vaccination. We found that antibody levels against WA1, Beta, and Omicron in serum from the Delta infection following vaccination were significantly higher than the Delta infection alone (Fig. 1e). In contrast, no significant difference was observed in the antibody titers against the Delta between the two groups (Fig. 1e). These findings revealed that the Delta infection following vaccination not only boosted high potency neutralizing antibodies against WA1 but also boosted antibody response to the Beta and Omicron variants regardless of CoronaVac or ZF2001.

\section{Discussion}

The rapid and widespread of the Omicron poses an urgent challenge to public health. Previous studies have shown that lower neutralizing titers have been associated with an increased risk of symptomatic COVID-19 ${ }^{19}$, indicating that completely lost or limited neutralizing activity against the Omicron may increase risk of infection and higher burden of disease ${ }^{20}$. Our study demonstrates significant resistance of the Omicron variant to serum neutralizing activity induced by the Delta infection only. However, despite the significant escape of the Omicron to antibody response, the recent two preprints have shown that the majority of $\mathrm{T}$ cell responses induced by infection or vaccination remain capable of recognizing the Omicron and previously emerged variants ${ }^{21,22}$. Whether cellular immunity will be effective as a secondlevel defense in preventing severe disease after Omicron infection in the absence of a potent neutralizing antibody response remains to be determined ${ }^{23,24}$.

Importantly, following vaccination, patients with the Delta infection effectively induced a substantial increase in serum neutralization against vaccine matched WA1 virus and the Beta ad Omicron variants. Given the similarity of the booster antigens for the infection with live virus and booster vaccination with mRNA, our findings are consistent with recent studies that a single dose mRNA in vaccinated individuals enhanced the ability to neutralize the Omicron ${ }^{8-11,25}$. Such enhanced neutralizing activity to variants may be due to the broad and potent neutralizing activity of antibodies produced by the evolved memory B cells were recruited into the plasma cell compartment ${ }^{26-28}$. However, higher levels of neutralizing serum activity might not necessarily prevent SARS-CoV-2 infection, as indicated by reports of Omicron infections in boostered individuals. Collectively, our data suggest that Delta infection following vaccination could generate a strong antibody response for previously vaccinated individuals, not only when considering the antibodies levels but also when examining results of the neutralizing activity to variants.

Limitations of our study include that the antibodies were measured about one month after the Delta infection in previously vaccinated individuals. Longitudinal follow-up will be required to determine the durability of the neutralizing antibody response to the Omicron. Our analysis was limited to fewer 
individuals receiving the ZF2001 vaccine. Measures of T and B cell responses can shed further light on whether Delta infection following vaccination might be sufficient for augmenting $T$ and $B$ cell memory against the Omicron.

In summary, Omicron causes widespread escape from neutralization by serum obtained the Delta infection only meaning that previously, Delta-infected individuals will have little protection from infection with Omicron. In contrast, the Delta infection following vaccination can induce robust neutralization against the immune evasive Omicron variant.

\section{Materials And Methods}

\section{Ethical Approval}

The study was approved by the Institutional Review Board of the Beijing Institute of Microbiology and Epidemiology (IRB number: AF/SC-08/02.60 and AF/SC-08/02.124). All enrolled participants provided written informed consent.

\section{Sera from patients with the Delta infection during a Delta outbreak}

Between October and November 2021, an outbreak of the Delta variant was identified, and 213 patients were reported, including 39 patients under the age of 18 . Among these patients, nearly all patients over 18 years old had received two doses of CoronaVac by Sinovac or three doses of ZF2001 (a recombinant tandem-repeat dimeric RBD-based protein subunit vaccine) by Anhui Zhifei Longcom, whereas nearly all patients under 18 years old had not received any vaccines. All patients had sequence confirmed Delta infection or PCR-confirmed symptomatic disease occurring whilst in isolation and in direct contact with Delta sequence-confirmed cases. At the time of discharging from the hospital, patients signed an informed consent to consent for the collection of data and blood sample.

\section{Sera from patients with the Delta infection in an imported cargo ship}

On April 23, 2021, a cargo ship departed from Xiamen Port in Fujian Province of China for repairing and maintaining at a dockyard in Zhoushan Port in Zhejiang Province, China, at which time 3 of $20 \mathrm{crew}$ members reported a fever. On April 24, a nasopharyngeal swab was collected from each crew member to screen SARS-CoV-2 infection. The nasopharyngeal swab samples from 12 members, including three members with fever, were tested positive for SARS-CoV-2 using real-time reverse transcription-polymerase chain reaction (RT-PCR) assay. Nasopharyngeal swabs from 2 patients with cycle threshold (Ct) values < 30 were sequenced for the whole viral genome of SARS-CoV-2 and were identified the variant B.1.617.2 (GISAID accession ID: EPI_ISL_3611059-3611060). Their serum samples were obtained between June and July 2021 after being discharged from the hospital, approximately 2 months after infection. 


\section{Pseudovirus production and neutralization assay}

Pseudovirus particles were generated as previously described ${ }^{29,30}$ by co-transfecting HEK-293T cells (ATCC, CRL-3216) with human immunodeficiency virus backbones expressing firefly luciferase (pNL4-3-RE-luciferase, provided by Dr. Lin-Qi Zhang from Tsinghua University) and pcDNA3.1 vector encoding either WA1 or mutated S proteins (Beta, Delta, and Omicron) plasmid. The medium was replaced with fresh medium at 24 hours, and supernatants were harvested at 48 hours post-transfection and clarified by centrifugation at $300 \mathrm{~g}$ for $10 \mathrm{~min}$ before aliquoting and storing at $-80^{\circ} \mathrm{C}$ until using. SARS-CoV-2 pseudovirus neutralization assay (pVNT) was performed as described ${ }^{31}$, with target cell line HeLa cells expressing ACE2 orthologs (HeLa-ACE2, kindly provided by Dr. Lin-Qi Zhang from Tsinghua University). Duplicate 3-fold 8-point serial dilutions (starting at 1:30) of heat-inactivated serum were incubated with 500-1000 TCID 50 of SARS-CoV-2 pseudotyped virus for 1 hour at $37^{\circ} \mathrm{C}$. HeLa-ACE2 (200,000 cells/well) were subsequently added into the mixture and incubated for approximately 48 hours at $37^{\circ} \mathrm{C}$ with $5 \%$ $\mathrm{CO}_{2}$. Luciferase activity was then measured using GloMax 96 Microplate Luminometer (Promega). The half-maximal neutralization titers for serum were determined by luciferase activity 48 hours after exposure to the virus-serum mixture with a four-parameter non-linear regression inhibitor curve in GraphPad Prism 8.4.1 (GraphPad Software). Titers are reported as the serum dilution that inhibited 50\% of infection ( $p V N T_{50}$ ). Sample with $\mathrm{pVNT}_{50}$ values no more than 30 (the detectable limit) was considered negative for neutralizing antibodies and were assigned to 10 for calculation.

\section{Statistical analysis}

Friedman test with false discovery rate method was used for multiple group comparisons. Wilcoxon ranksum test was used to analyze the difference between the two groups. All statistical analyses were performed using GraphPad Prism (version 8.4.2, La Jolla, California, USA), and all statistical tests were 2sided with a significance level of 0.05 .

\section{Declarations}

\section{Acknowledgments}

We thank all study subjects for their participation in our studies. This work was supported by grants from the Beijing Natural Science Foundation (L202038) and the Natural Science Foundation of China (81773494 and 81621005).

\section{Author contributions}


MJM conceived the study. HXG, JR, LY, ZY, PJ, and EHD collected serum samples. LY and LKZ performed serology assays. LY, LKZ, and MJM analyzed the data; MJM drafted the manuscript. All authors reviewed and approved the final manuscript.

\section{Declaration of interests}

We declare no competing interests.

\section{References}

1. Wang, P., et al. Antibody resistance of SARS-CoV-2 variants B.1.351 and B.1.1.7. Nature 593, 130135 (2021).

2. Abu-Raddad, L.J., Chemaitelly, H. \& Butt, A.A. Effectiveness of the BNT162b2 Covid-19 Vaccine against the B.1.1.7 and B.1.351 Variants. N Engl J Med 385, 187-189 (2021).

3. Madhi, S.A., et al. Efficacy of the ChAdOx1 nCoV-19 Covid-19 Vaccine against the B.1.351 Variant. N Engl J Med 384, 1885-1898 (2021).

4. Sadoff, J., et al. Safety and Efficacy of Single-Dose Ad26.COV2.S Vaccine against Covid-19. N Engl J Med 384, 2187-2201 (2021).

5. WHO. Weekly epidemiological update on COVID-19 - 11 May 2021. Vol. 2021 (2021).

6. Mlcochova, P., et al. SARS-CoV-2 B.1.617.2 Delta variant replication and immune evasion. Nature 599, 114-119 (2021).

7. Viana, R., et al. Rapid epidemic expansion of the SARS-CoV-2 Omicron variant in southern Africa. Nature (2021).

8. Garcia-Beltran, W.F., et al. mRNA-based COVID-19 vaccine boosters induce neutralizing immunity against SARS-CoV-2 Omicron variant. Cell (2022).

9. Henning, G., et al. mRNA booster immunization elicits potent neutralizing serum activity against the SARS-CoV-2 Omicron variant. Nature Medicine (2022).

10. Hoffmann, M., et al. The Omicron variant is highly resistant against antibody-mediated neutralization - implications for control of the COVID-19 pandemic. Cell(2021).

11. Planas, D., et al. Considerable escape of SARS-CoV-2 Omicron to antibody neutralization. Nature (2021). 
12. Liu, L., et al. Striking Antibody Evasion Manifested by the Omicron Variant of SARS-CoV-2. Nature (2021).

13. Cao, Y., et al. Omicron escapes the majority of existing SARS-CoV-2 neutralizing antibodies. Nature (2021).

14. Tang, P., et al. BNT162b2 and mRNA-1273 COVID-19 vaccine effectiveness against the SARS-CoV2 Delta variant in Qatar. Nature Medicine 27, 2136-2143 (2021).

15. Eyre, D.W., et al. Effect of Covid-19 Vaccination on Transmission of Alpha and Delta Variants. $N$ Engl J Med (2022).

16. Nanduri, S., et al. Effectiveness of Pfizer-BioNTech and Moderna Vaccines in Preventing SARSCoV-2 Infection Among Nursing Home Residents Before and During Widespread Circulation of the SARSCoV-2 B.1.617.2 (Delta) Variant - National Healthcare Safety Network, March 1-August 1, 2021. MMWR Morb Mortal Wkly Rep 70, 1163-1166 (2021).

17. Kislaya, I., et al. Comparative Effectiveness of Coronavirus Vaccine in Preventing Breakthrough Infections among Vaccinated Persons Infected with Delta and Alpha Variants. Emerg Infect Dis 28(2021).

18. Sholukh, A.M., et al. Evaluation of Cell-Based and Surrogate SARS-CoV-2 Neutralization Assays. $J$ Clin Microbiol 59, e0052721 (2021).

19. Khoury, D.S., et al. Neutralizing antibody levels are highly predictive of immune protection from symptomatic SARS-CoV-2 infection. Nat Med 27, 1205-1211 (2021).

20. Rössler, A., Riepler, L., Bante, D., Laer, D.v. \& Kimpel, J. SARS-CoV-2 B.1.1.529 variant (Omicron) evades neutralization by sera from vaccinated and convalescent individuals. medRxiv, 2021.2012.2008.21267491 (2021).

21. Keeton, R., et al. SARS-CoV-2 spike T cell responses induced upon vaccination or infection remain robust against Omicron. medRxiv, 2021.2012.2026.21268380 (2021).

22. Tarke, A., et al. SARS-CoV-2 vaccination induces immunological memory able to cross-recognize variants from Alpha to Omicron. bioRxiv (2021).

23. Sette, A. \& Crotty, S. Adaptive immunity to SARS-CoV-2 and COVID-19. Cell 184, 861-880 (2021).

24. Goel, R.R., et al. mRNA vaccines induce durable immune memory to SARS-CoV-2 and variants of concern. Science 374, abm0829 (2021).

25. Carreño, J.M., et al. Activity of convalescent and vaccine serum against SARS-CoV-2 Omicron. Nature (2021). 
26. Stamatatos, L., et al. mRNA vaccination boosts cross-variant neutralizing antibodies elicited by SARS-CoV-2 infection. Science(2021).

27. Greaney, A.J., et al. Antibodies elicited by mRNA-1273 vaccination bind more broadly to the receptor binding domain than do those from SARS-CoV-2 infection. Science translational medicine 13(2021).

28. Turner, J.S., et al. SARS-CoV-2 mRNA vaccines induce persistent human germinal centre responses. Nature 596, 109-113 (2021).

29. Nie, J., et al. Quantification of SARS-CoV-2 neutralizing antibody by a pseudotyped virus-based assay. Nature protocols 15, 3699-3715 (2020).

30. Li, Q., et al. The Impact of Mutations in SARS-CoV-2 Spike on Viral Infectivity and Antigenicity. Cell 182, 1284-1294 e1289 (2020).

31. Nie, J., et al. Establishment and validation of a pseudovirus neutralization assay for SARS-CoV-2. Emerg Microbes Infect 9, 680-686 (2020).

\section{Tables}

Table 1. Characteristics of the enrolled patients infected with SARS-CoV-2 Delta infection following vaccination and Delta infection only in Shijiazhuang, China. 


\begin{tabular}{|c|c|c|}
\hline \multirow[t]{2}{*}{ Characteristics } & \multicolumn{2}{|l|}{ Patients } \\
\hline & $\begin{array}{l}\text { Delta infection following } \\
\text { vaccination }\end{array}$ & $\begin{array}{l}\text { Delta infection } \\
\text { only }\end{array}$ \\
\hline Age (median, IQR) & $42.0(37.0-65.0)$ & $10.0(8.0-12.0)$ \\
\hline \multicolumn{3}{|l|}{ Age group (\%) } \\
\hline$>18$ & $29(93.5)$ & $2(7.1)$ \\
\hline$\leq 18$ & $2(6.5)$ & $26(92.9)$ \\
\hline \multicolumn{3}{|l|}{ Sex (\%) } \\
\hline Male & $16(51.6)$ & $14(50.0)$ \\
\hline Female & $15(48.4)$ & $14(50.0)$ \\
\hline \multicolumn{3}{|l|}{ Disease severity (\%) } \\
\hline Asymptomatic & $2(6.5)$ & 0 \\
\hline Mild & $7(22.6)$ & $22(78.6)$ \\
\hline Moderate & $21(67.7)$ & $6(21.4)$ \\
\hline Severe & $1(3.2)$ & 0 \\
\hline \multicolumn{3}{|l|}{ Vaccination status (\%) } \\
\hline Yes & $31(100)$ & 0 \\
\hline No & 0 & $28(100)$ \\
\hline \multicolumn{3}{|l|}{ Type of vaccine (\%) } \\
\hline CoronaVac & $22(71.0)$ & 0 \\
\hline ZF2001 & $9(29.0)$ & 0 \\
\hline $\begin{array}{l}\text { Interval between } 2^{\text {nd }} \text { or } 3^{\text {rd }} \text { dose and symptom } \\
\text { onset (median, IQR) }\end{array}$ & $118.0(47.0-148.0)$ & NA \\
\hline $\begin{array}{l}\text { Interval between symptom onset and sampling } \\
\text { (median, IQR) }\end{array}$ & $47.0(36.0-53.0)$ & $\begin{array}{l}38.0(31.5- \\
45.8)\end{array}$ \\
\hline
\end{tabular}

IQR, interquartile range; NA, not available. 
Table 2. Characteristics of imported patients with SARS-CoV-2 Delta infection only in a cargo ship in Zhoushan, China.

\begin{tabular}{|ll|}
\hline Characteristics & Patient with the Delta infection only \\
\hline Age (median, IQR) & $37.0(29.3-45.5)$ \\
\hline Sex (\%) & $8(100)$ \\
\hline Male & \\
\hline Disease severity (\%) & $6(75.0)$ \\
\hline Moderate & $2(25.0)$ \\
\hline Severe & \\
\hline Vaccination status (\%) & 0 \\
\hline Yes & $8(100 \%)$ \\
\hline No & $53.0(53.0-87.0)$ \\
\hline Interval between symptom onset and sampling (median, IQR) & \\
\hline
\end{tabular}

IQR, interquartile range.

\section{Figures}



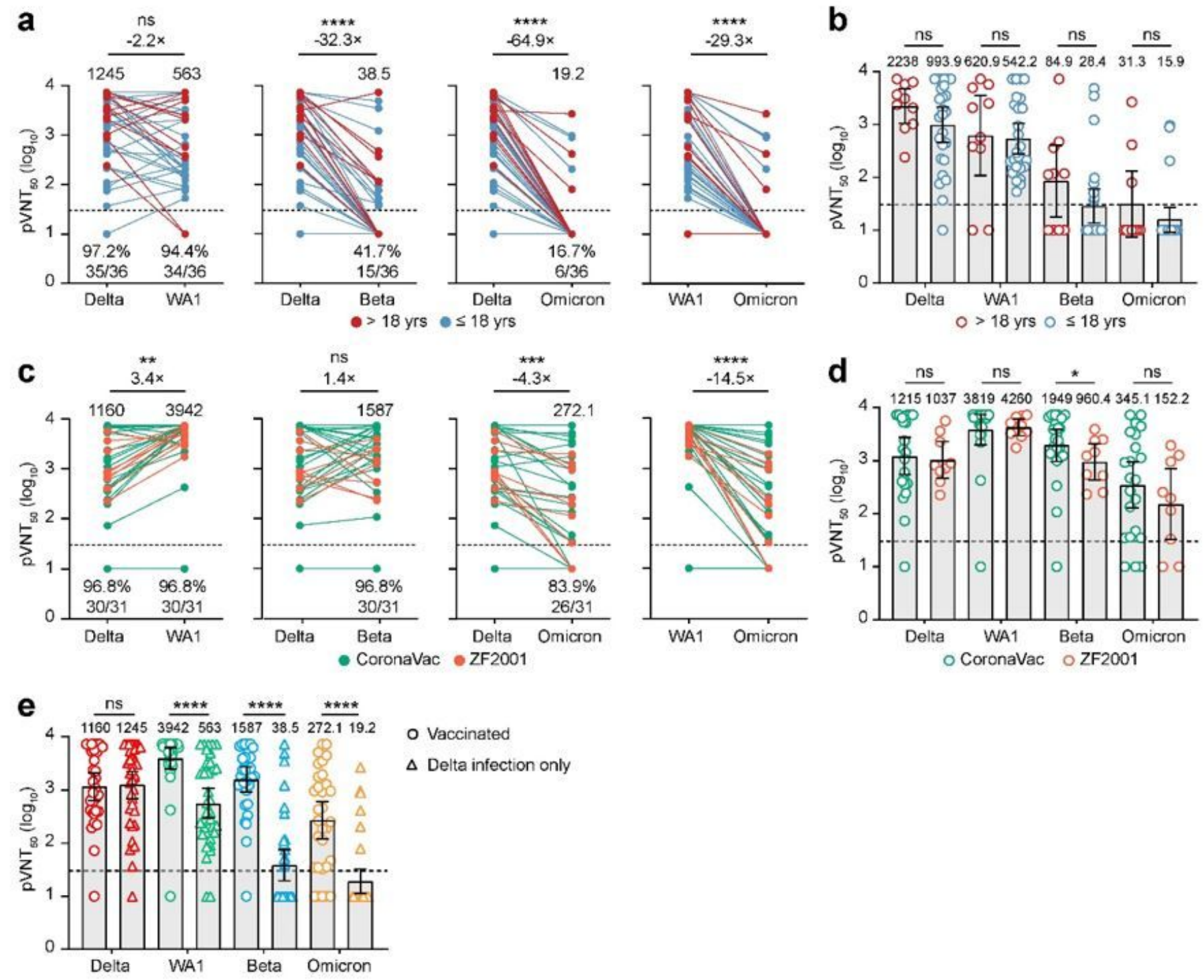

o Vaccinated

$\Delta$ Delta infection only

\section{Figure 1}

Neutralization of Delta, WA1, Beta, and Omicron to sera from convalescent patients with Delta infection following vaccination and Delta infection only. a. Neutralizing activity of sera from convalescent patients with Delta infection only $(n=36)$ sampled about one month after infection. Participants were either $>18$ years old (red, $n=10$ ) or less than 18 years old (blue, $n=26$ ). b. Comparison of the antibody titer between patients $>18$ years old and patients $\leq 18$ years old. c. Neutralizing activity of sera from convalescent patients with Delta infection following vaccination $(n=31)$ sampled about one month after infection. Participants were either received CoronaVac (green, $n=22$ ) or ZF2001 (orange, $n=9$ ). d. Comparison of the antibody titer between patients vaccinated CoronaVac $(n=22)$ and ZF2001 $(n=9)$. e. Comparison of the antibody titer between vaccinated patients and Delta infection only by Delta (red), WA1 (green), Beta (blue), and Omicron (Orange). The neutralization titers of the sera against the indicated viral variants are expressed as $\mathrm{pVNT}_{50}$ (50\% pseudovirus inhibitory dilution). Geometric mean titers (GMT) are shown above each column. The percentage of samples with neutralizing activity in $\mathrm{pVNT}_{50}$ are shown below each column. The heights of bars indicate GMT; error bars show $95 \%$ confidence intervals. The horizontal 
dotted line represents the limit of detection at 1:30. A two-tailed Friedman test with a false discovery rate for multiple comparisons was performed to compare WA1, Beta, and Omicron to the Delta in $\mathbf{a}$ and $\mathbf{c}$ as well as Wilcoxon rank-sum test was performed in $\mathbf{b}$, $\mathbf{d}$, and $\mathbf{e}$. ${ }^{*} p<0.05, * * p<0.01, * \star * p<0.001$, and $* \star \star *$ $\mathrm{p}<0.0001$. ns, not significant.

\section{Supplementary Files}

This is a list of supplementary files associated with this preprint. Click to download.

- ReportingSummary.pdf

- EditorialPolicyChecklist.pdf 